\title{
A viscosity-mediated model for relating the gloss and film thickness of coatings
}

Julio Uribe-Padilla $\left({ }^{*}\right)^{a, b,{ }^{, *}}$; Moises Graells-Sobre ${ }^{b}$; Juan Salgado-Valle ${ }^{a}$; Julia López ${ }^{a}$

a-Chemical Engineering Department, Universitat Politècnica de Catalunya (UPC), EEBE, 08019, Av. Eduard Maristany 10-14, Barcelona, Spain.

b-Polyurethane R \& D Department, BASF Española S.L., Curtex Division, 08907, Carretera del Mig, 219, Barcelona, Spain.

๑- Currently at Production Department, Sumitomo Bakelite Europe Barcelona (SBHPP), SBEB, 08170, Gran Vial 4, Barcelona, Spain.

Highlights

- Film thickness and gloss have been correlated for several glossy and matt coatings up to moderately thick layers.

- The model contemplates the hindering of film formation due to non-Newtonian behaviour as well as the presence and concentration of matting agents.

- A characteristic film-formation related time has been correlated to the levelling time.

- On this basis, a viscosity-film thickness relationship has been developed and validated.

\section{Abstract}

Film thickness determines many key coating properties including gloss. Current literature hardly addresses the development of methods to directly correlate film thickness with gloss. Thus, the lack of accurate models hinders further product design and optimisation of coating products and processes. In this contribution, a previously derived glossviscosity relationship is turned into a gloss-film thickness mathematical model. Experimental results of both matting and glossy agents are found to be successfully predicted by a model that is revealed as a simple and useful tool for day-to-day calculations.

Keywords: Film thickness, gloss, matt, optical properties, coatings, mathematical modeling 
2 It has been recently reported that the gloss of a thickened-to-application hybrid 3 polyurethane dispersion (PUD) with matting purposes can be correlated with viscosity

4 [1]. These studies fostered revisiting the influence of other coating-relevant variables on

5 gloss [2], [3]. Special interest is now paid to film-thickness (FT or $\delta$ ) and how it affects

6 optical properties and, in particular, matting efficiency. All coating manufacturers are

7 aware of the fact that FT is a key parameter not only from an aesthetic but also from a

8 practical point of view. For instance, the total cost (operational + raw materials) per unit

9 area of a coating process $\left(C_{c}\right)$ is directly proportional to FT (i.e. $C_{c} \propto \frac{\bar{\delta} \cdot \rho}{Y_{t} \cdot w}$ ) being $\bar{\delta} \cdot \rho=$

$10 \Gamma_{c}$ the total coating amount per unit area and $Y_{t} \cdot w$ the total transference yield per

11 solid content [4]. Furthermore, recent advances in coating studies have shown that FT

12 is also of paramour importance in electrical [5], [6], thermal [7], [8], mechanical [9]-

13 [11] or barrier properties [12]-[14].

14 Current chemical engineering trends ask for practical predictive models supporting decision-making procedures and enhancing current and future product and process design [15]-[17]. Past academic research concerning coatings' industry is based in

17 complex momentum balance problem formulations. Such approaches are computationally expensive and require a deep understanding of transport-phenomena, solving the Navier-Stokes equations for non-Newtonian fluids while taking into account thermal effects and chemical reactions [18]-[27]. When tackling the optical properties of coatings a similar situation is faced and intricate physics (like photonics, nonlinear optics, etc.) must be dealt with [28]-[30]. Thus, an affordable approach combining film 
23 thickness and gloss would be desirable for practical day-to-day calculations and

24 decision-making.

25 Therefore, the aims and contributions of this work are to:

26 - Derive a simple mathematical model that relates gloss and film thickness.

27 - Assess its usefulness and validity-limitations specially focusing on testing 28 different coatings and verifying functional dependences.

29

30

\section{2-Modeling approach}

\section{1-Hypothesis}

Let the following assumptions hold:

- The coating is well-represented by a prismatic geometry with its volume being proportional to $\delta^{3}$.

- The application system is a roller blade-conveyor belt-like system.

- The relation between viscosity and FT can be conveniently represented by some power law.

- FT is the most relevant dimension of the coating under study (i.e. final effects are neglected [4]).

- Hiding-power related effects are only relevant below a minimum FT.

- The process is isothermal and no chemical interaction occurs between the coating and the substrate nor the atmosphere.

- The system shows anisotropic behaviour.

- The liquid coating is or behaves like a Newtonian fluid for a sufficiently wide shear rate interval. 
- Average film thickness accounts for roughness and other surface texture properties.

- No micro-sagging or micro-bubbling occurs.

\section{2-Mathematical model}

The problem addressed in this paper deals with how FT affects the final gloss of a given coating. If a suitable substrate ( with gloss $g_{s}$ ) is to be coated up to a matting degree of $g_{c}$, it is desirable that $g_{c} \ll g_{s}$. The evolution of a liquid coated film when readily applied on such substrate and left to dry a certain amount of time $\Delta t$ is illustrated in



55 Fig 1: Film formation dynamics involved in the drying of a liquid film

56 Along the drying process, the mass of the film $(\mathrm{m})$ and the associated matting content ${ }^{1}$

57 (w) need to satisfy the mass balance:

$$
m_{0} w_{0}=m_{f} w_{f}
$$

58 When all the solvent is evaporated $w_{f}=1, m_{f}=m_{0} w_{0}$ and a thinner coat is obtained.

59 Assuming a prismatic geometry the total volume of the film $(\mathrm{V})$ is the product of film 60 surface (S) and FT:

\footnotetext{
${ }^{1}$ More specifically $w=w_{\text {Polymer }}+w_{M A}$ or $w=\xi w_{M A}$ where $\xi=\frac{w_{\text {Polymer }}}{w_{M A}}-1$. This specification is relevant when model parameters are to be correlated specifically with matting agent concentration.
} 


$$
V=S \delta
$$

61 A real film will not have a perfectly smooth surface (Fig.2) because FT is generally

62 inhomogeneous and varies both with length ( $x$ direction) and width ( $y$ direction). Hence

63 surficial area must be calculated from roughness data from height (z direction) [31]:

$$
S=\iint_{f i l m} \delta(x, y) d x d y
$$

64 Since we are studying the effect of FT all geometric expressions should depend solely on obtained after evaluating the integral all over the film.

$$
S=\left(\delta \int_{0}^{\text {length }} \Phi_{\mathrm{x}}(x) d x\right) \cdot\left(\delta \int_{0}^{\text {width }} \Phi_{\mathrm{y}}(y) d y\right)=\varphi_{x} \varphi_{y} \delta^{2}=\varphi \delta^{2}
$$

68 Combining Eq.2 with Eq.4 it can be seen that the volume of the film is proportional to $69 \delta^{3}$.

$$
V=S \delta=\varphi \delta^{3}
$$



The coating differential does not have constant film-thickness but it is smooth enought for assuming that $\frac{d \delta}{d x}=\frac{d \delta}{d y}=0$ hence $\delta(x, y)=\delta$

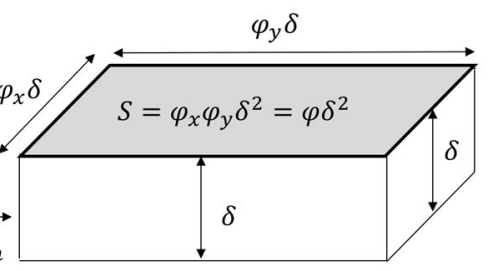

Geometrically equivalent film with normalized surface area $S=A_{\text {film }}$ which has a total volume $V \propto \delta^{3}$

Fig 2: Mathematical modeling of the film's surface in which a real film is transformed in an equivalent smooth-surface film with identical surface. compute the following density ratio: 


$$
\frac{\rho_{0}}{\rho_{f}}=A_{T}\left(\frac{\delta_{f}}{\delta_{0}}\right)^{3}
$$

75 Eq.6 is useful given the difficulties in measuring near-to-solid coating bulk properties

$76[32] . A_{T}$ is a weight-fraction modified normalizing factor defined as:

$$
A_{T}=\frac{\varphi_{f} w_{f}}{\varphi_{0} w_{0}}
$$

77 Several authors [33]-[38] have proposed film thickness-viscosity relationships (FTVRs)

78 that should follow a power-like model:

$$
\mu \propto \delta^{n}
$$

79 Nonetheless, intense debate is still found nowadays regarding $n$ 's value. In general, $n$ can be found to fall between 1.43-1.50 (closer to theoretical predictions mostly based on Landau-Levich theory [39], [40]) and 2.00 (verifying most empirical observations and simpler modeling strategies [41]). A remarkable exception is that of asphalts [42], cements [43] and bituminous substances [44] for which $n$ can be either positive or negative meaning that viscosity can decrease or increase with $\mathrm{FT}^{2}$.

85 Since this work is aimed at developing a practical tool FTVR will be used with $n=2$. The simplest model for which $\mu \propto \delta^{2}$ is derived from a force balance where capillary forces match viscous forces. Despite this, an alternative reasoning (Eq.9 to Eq.15) based on film-forming dynamics is proposed here, giving a new FTVR with $n=2$.

During film formation, both surficial and internal stresses will appear due to a myriad of causes [45], [46]. These momentum transports (Fig. 1) can experience transitions that are here modelled by a characteristic periods $\lambda^{*}$ [47], [48]. Letting $\ell$ be a characteristic

\footnotetext{
2 The value of $\mathrm{n}$ is also highly technology-dependent being the $1.43 \leq n \leq 2$ values found in conveyor-belt like systems. Conversely wide range of processes carried out in continuous spinning disk reactors (SPR's) follow FTVRs with $2.8<n<3.2$. The effect of solvents also conditions final FT in drying films [72] .
} 
92 dimension of the system it is possible to correlate it with $\lambda^{*}$ by means of viscosity and

93 density. Furthermore if $\ell=\delta$ then:

$$
\lambda^{*}=\frac{\rho \ell^{2}}{\mu} \rightarrow \lambda^{*}=\frac{\rho \delta^{2}}{\mu}
$$

94 Along the film formation process several characteristic times can be of interest. The

95 model focuses on the initial and final states represented in the mass balance $\left(\lambda_{0}^{*}\right.$ and $\lambda_{f}^{*}$,

96 respectively), which are here considered proportional:

$$
\lambda_{0}^{*}=\alpha \lambda_{f}^{*}
$$

97 where $0<\alpha<1$. Developing Eq.10 and introducing the definition of $\lambda$ yields :

$$
\frac{\rho_{0}}{\rho_{f}}=\alpha\left(\frac{\mu_{0}}{\mu_{f}}\right)\left(\frac{\delta_{f}}{\delta_{0}}\right)^{2}
$$

98 Rearranging Eq.11 a quadratic expression is obtained:

$$
\mu_{f}=\alpha^{\prime} \delta_{f}^{2}
$$

99 Where $\alpha^{\prime}$ is:

$$
\alpha^{\prime}=\alpha \frac{\mu_{0} \rho_{f}}{\delta_{0}^{2} \rho_{0}}
$$

100 It can be shown that $\alpha^{\prime}$ includes time-related information giving higher credit to the 101 modelling approach. In particular, the levelling time $\lambda_{\text {lev }}$ (Eq.14) is invoked at this point 102 as defined from Orchard's theory [49]-[51]. $\lambda_{\text {lev }}$ is directly proportional to viscosity and 103 inversely proportional to surface tension :

$$
\lambda_{\text {lev }} \propto \frac{\mu}{\sigma}
$$

104 From Eq.14 two conclusions can be drawn:

105 - The more viscous the coating the more time it takes for its film to flatten, hence remaining thicker. 
108 The proportionality factor between $\lambda_{\text {lev }}$ and $\frac{\mu}{\sigma}$ can be taken as an equivalent average

109 film thickness $(\bar{\delta})^{4}$. Considering also the Ohnesorge number $\left(O h=\frac{\mu}{\sqrt{\rho \sigma \bar{\delta}}}\right)$ the

110 proportionality factor can now be defined as a function of $\lambda_{l e v}$ :

$$
\frac{\mu}{\bar{\delta}^{2}}=\frac{\rho}{\lambda_{\text {Lev }}} O h^{2} \rightarrow \alpha^{\prime}=\frac{\rho}{\lambda_{\text {Lev }}} O h^{2}
$$

111 Further mathematical treatment of the first part of Eq. 15 gives an interesting

112 nondimensional expression (Eq. 16):

$$
\sqrt{\frac{C a}{B o}}=O h
$$

where Ca and Bo respectively stand for the Capillarity [54] and Bond numbers. Such equation defines a working space [55] which characterizes the coating process 5 .

116 Finally, combining Eq.6 with Eq.11 and invoking the definition of $R=\frac{\mu_{0}}{\mu_{f}}$ leads to:

$$
R=\frac{1}{\alpha} A_{T}\left(\frac{\delta_{f}}{\delta_{0}}\right)
$$

117 This definition of $R$ allows connecting both gloss and FT by means of viscosity. Providing

118 that DFT is significantly larger than average particle size, it seems intuitive to state that

119 the thicker the film the glossier it will be ${ }^{6}$. Choosing a previously derived model for traditional silica-based MA [1]:

$$
g=g_{0} R^{t}
$$

121 and combining it with Eq.16 a gloss-film thickness relationship (GFTR) is obtained.

\footnotetext{
3 This might seem counterintuitive since surface tension is a measure of the degree of substrate-coating affinity but this particular issue is thoroughly covered in literature .

4 The proportionality factor between $\lambda_{\text {lev }}$ and $\frac{\mu}{\sigma}$ is $\frac{3 f_{z}^{4}}{(2 \pi)^{4} h^{3}}$ and has equivalent length dimensions. It includes the ratio between film's striation degree $\left(f_{Z}\right)$ and a film thickness that includes roughness $(h)$ [52]-[53]. Taking into account this, $\frac{3 f_{Z}^{4}}{(2 \pi)^{4} h^{3}}=\bar{\delta}$.

${ }^{5}$ Further considerations about this result would be worth noting but are out of the scope of the present paper.

${ }^{6}$ A thicker layer will mask substrate's effects and defects, yielding a smoother surface and hence, a glossier one. This is also In good agreement with leveling time since coatings with higher viscosity are glossier [1].
} 


$$
g=\mathcal{B} \delta_{f}^{t}
$$

122 In which $\mathcal{B}$. Can be defined as:

$$
\mathcal{B}=g_{0}\left(\frac{A_{T}}{\alpha \delta_{0}}\right)^{t}
$$

123 This expression confirms the general behaviour expected (gloss increases with FT).

124 Despite this, two questions arise concerning the case where no MA is applied at all and

125 if it adequately represents what happens for increasingly thicker layers. Regarding the

126 first one, a condition for the no-coating border (NCBC) must be defined:

$$
\delta_{f}=0 \rightarrow g=g_{s}
$$

127 When no coating is applied, the recorded gloss must be that of the substrate. Once a

128 layer of product starts to deposit onto the substrate, the gloss readings start to change.

129 For thicknesses near or below average particle size, the coating's hiding power is

130 impoverished. Then, since no good substrate coverage is obtained, the matting

131 efficiency will be substantially lowered until a minimum film thickness is attained. Such

132 statement is in good agreement with early findings [56]. Going beyond the so-called

133 "optimum ${ }^{7}$ " film thickness $\left(\delta_{c}^{*}\right)$ which leads to minimum gloss [57] spurs some

\footnotetext{
7 The optimum label is misleading though and, far from being a desirable situation, only consists of the minimum FT required for ensuring sufficient coverage [58] but without satisfying other requirements or design constraints. For instance, haptics and other surface texture indicators [59] are often not met when $\delta=\delta_{c}^{*}$.
} 


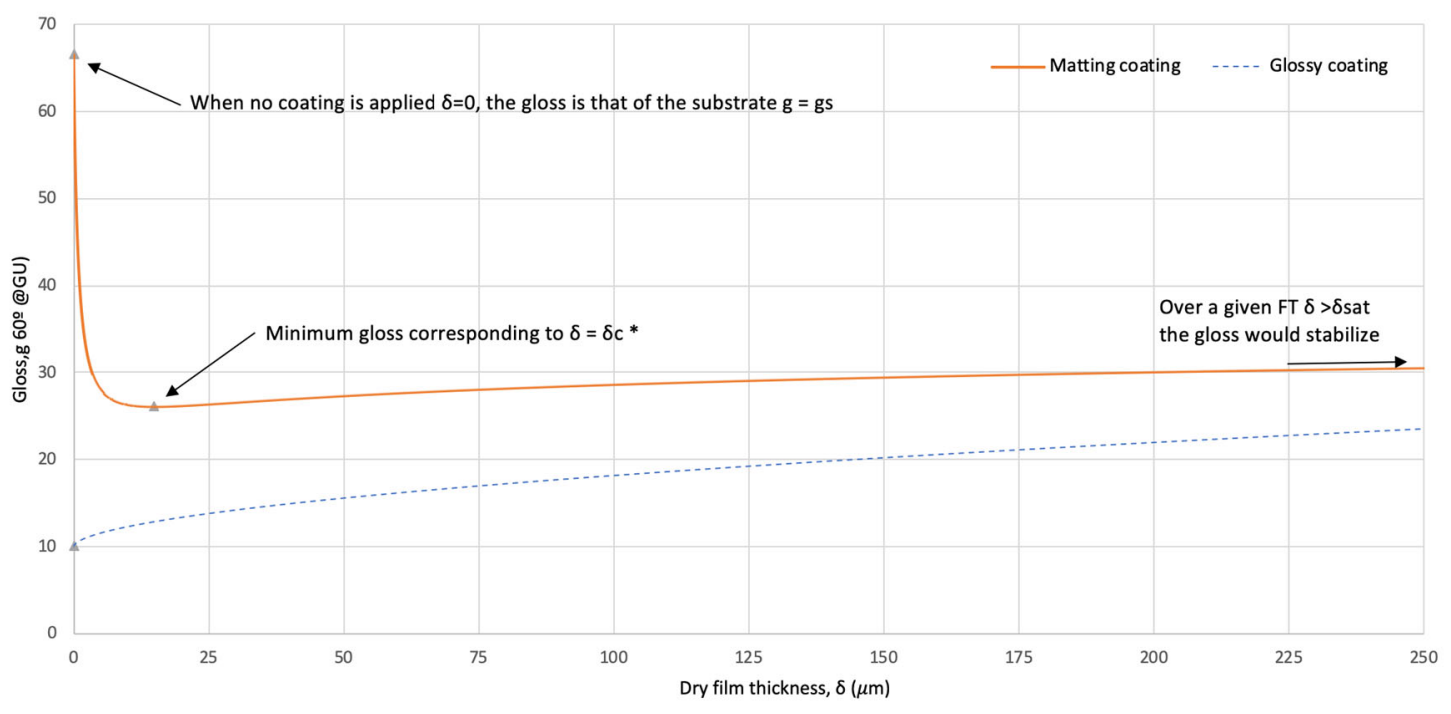

136 Fig. 3: Qualitative graph showing a sample for a matting and a glossy coating up to $\delta \leq$

$137 \delta_{\text {sat }}$. The grey triangles show specific data points of interest.

138 The work of Fletcher [60] introduced two effectiveness factors respectively related to 139 gloss $\left(g_{e f f}\right)$ and viscosity $\left(\mu_{e f f}\right)$. Both parameters were involved in the derivation of

140 two complementary gloss-concentration and viscosity-concentration models.

141 Whilst (for the traditional MA) $\mu_{\text {eff }}$ can be related to the intrinsic viscosity according 142 the Krieger-Dougherty's theory [61], $g_{\text {eff }}$ lacks of physical meaning. Further 143 experimentation for various MAs at different concentrations showed that $g_{\text {eff }}$ 144 decreased with FT ( $\delta>20 \mu m)$ but a general trend could not be devised.

145 In the previous derivation for the same MA [1], $g_{\text {eff }}$ and $\mu_{\text {eff }}$ where correspondingly 146 rearranged as $g_{e f f}=\ln \left(t_{1}\right)$ and $\mu_{e f f}=1 / t_{2}$. Now, using the gloss-rheology model for

147 the hybrid MA and recalling the expression of $R$ found in Eq.16, Eq.21 is obtained:

$$
\frac{g}{g_{0}}=1+t_{1}\left(\wp \delta^{t_{2}}-1\right)
$$


148 where $\wp=\left(\frac{A_{T}}{\alpha \delta_{0}}\right)^{t_{2}}$. Note that $\wp$ is expected to be $\mathcal{O}_{\wp}\left(10^{-1}\right)$ since average $\mathrm{R}$ was $149 \mathcal{O}_{\wp}\left(10^{-3}\right)$. Moreover, $g_{\text {eff }}$ is affected by FT [60] and consequently $t_{1}$ will change 150 somehow with FT as well. Rearranging Eq.21, two different parts can be identified:

$$
g=\underbrace{g_{0}\left(1-t_{1}\right)}_{\text {Part } A}+\underbrace{t_{1} g_{0} \wp \delta^{t_{2}}}_{\text {Part } B}
$$

151 For a given FT the gloss of the coating will be affected by-hiding power (Part A) or 152 unaffected by it (Part B). This is reasonable taking into account Fig.3 and also with 153 previous finding of the role of $t_{1}$ in maximum attainable gloss depletion [1].

154 Gloss can be directly related to the fraction of reflected light (reflectivity, r) [62], which 155 in turn is related to some scattering coefficient $\left(P_{\Psi}^{\infty}\right)$ able of effectively promoting 156 matting properties. Though the work of Fletcher [60] did not correlated $g_{\text {eff }}$ with $P_{\Psi}^{\infty}$ 157 or $r$ it indeed indicated that $g_{\text {eff }}$ depended on a diffuse response to illumination. 158 Furthermore, assuming $t_{2}$ remains constant, $t_{1}$ is likely of being more sensitive to FT 159 when $\mathrm{FT}<\delta_{c}^{*}$, while gradually becoming less influenced by FT when FT $\geq \delta_{c}^{*}$ [63]. Hence, 160 a reasonable hypothesis is that $t_{1}$ could be modeled as a (hyperbolic) trigonometric 161 function.

$$
t_{1}=f_{1}\left(g_{\text {eff }}\right)=f_{2}\left(P_{\Psi}^{\infty}\right)=f_{3}(r)=\left(\begin{array}{c}
\tanh ^{-1} \\
\operatorname{coth}^{-1} \\
\ldots
\end{array}\right)(\delta)
$$

162 This hypothesis is consistent with the Kubelka-Munk theory of reflectance and will be 163 discussed later in the corresponding section. Until then, this unknown function of FT will 164 be denoted as $J(\delta)$. In order to validate this last reasoning (Eq. 13) $t_{1}$ is now set 165 constant. Then, differentiating Eq.12 and further integrating using the NCBC, Eq. 24 is obtained: 


$$
g=g_{s}+t_{1} \wp \delta^{t_{2}}
$$

167 Note now that Eq.24 represents the expected behaviour of a high-gloss coating applied 168 to a substrate of gloss $g_{s}$ as previously reported [65]. The fact that scattering coefficient

169 does not depend on FT (i.e. $\left.\frac{\partial t_{1}}{\partial \delta}=0\right)$ was already established for glossy substances 170 such as white alkyds, glossy latexes and glossy paints [66].

171 Additionally, recalling that $1.43 \leq \mathrm{n} \leq 2$ it is expectable that $0.50 \leq \mathrm{t}_{2} \leq 0.70$ since

172 they are inversely proportional.Then, $t_{1}$ can be expressed in different ways depending 173 on the kind of coating under consideration.

$$
t_{1}=f_{1}\left(g_{e f f}\right)=f_{2}\left(P_{\Psi}\right)= \begin{cases}\text { Glossy } & \frac{\partial t_{1}}{\partial \delta}=0 \\ M a t t & J(\delta)\end{cases}
$$

174 In conclusion:

$$
\begin{array}{ll}
\text { Glossy } & \frac{g}{g_{0}}=1+t_{1}\left(\wp \delta^{t_{2}}-1\right) \\
\text { Matt } & \frac{g}{g_{0}}=1+J(\delta)\left(\wp \delta^{t_{2}}-1\right)
\end{array}
$$

\section{3-Experimental material and methods}

177 This study has analysed experimental data from the literature and from freshly 178 produced PUDs samples obtained from BASF's production site in L'Hospitalet de 179 Llobregat in Catalonia (Spain). The samples from BASF were used as received.

\section{1-Polymer coatings}

181 6-10 g of liquid sample where transferred via syringe to a standardized drawdown cardboard (WP-1, LENETA, New Jersey-United States). The card was secured to an application support bed. Several helical bar manual coaters from 10 to $100 \mu \mathrm{m}$ of wet FT (NEUTREK, Eibar-Spain) where used for preparing the polymer films at an extension 
speed of approximately $42 \mathrm{~mm} \cdot \mathrm{s}^{-1}$. The polymer film was then dried on a clean and flat glass plate in a preheated air laboratory oven (ED115, Binder GmbH, Bohemia-Germany)

187 keeping it at $60^{\circ} \mathrm{C}$ during 10 minutes. After being taken out of the oven the samples 188 where left to cool to room temperature on a flat and clean surface during $1 \mathrm{~h}$.

\section{2-Film thickness measurements}

190 Film thickness was determined from the same polymer-coated black drawdown 191 cardboards (DCBs) used for gloss testing by means of an Etalon Microrapid 226 (Brown

\begin{tabular}{|c|c|c|}
\hline Coating & $192 \delta$ & \& Sharpe, Sweden). The actual polymer FT \\
\hline Substrate (DCB) & $\Delta \delta \quad 193 \delta_{S}$ & was determined by subtracting a blank \\
\hline
\end{tabular}

194 value (corresponding to the $\mathrm{DCB}$ ) to the total thickness measured. A linear relationship 195 between wet and dried FTs, would be expected, indicating proper drying and uniform film deposition. This was already implied in Eq.6 which also predicted higher dried FTs

197 for solid-rich liquid coatings (see Eq. 27 for the case where $\varphi_{0}=\varphi_{f}, \rho_{0}=\rho_{f}$ and 198 completely dried film).

$$
\begin{aligned}
& \delta_{f}=\Delta \delta_{D}-\delta_{s} \\
& \delta_{f}=\sqrt[3]{w_{0}} \cdot \delta_{0}
\end{aligned}
$$

199

200 The value of $\delta_{s}$ is obtained for each coating extension test as the average value of 10 201 random measurements alongside the whole available extension surface.

202 Special care was taken in not damaging the film extension as gloss measurements where 203 performed before FT measurements. Absence of film damage was inspected both 204 visually (by two of the authors) and by means of a digital microscope (Swift digital M10TBTW1, China). 
207 The gloss of each sample was randomly recorded at least 10 times with a glossmeter 208 (REFO 3, HACH, Manchester-United Kingdom) according to UNE-EN-ISO 2813:1999. The 209 measurements where performed varying orientation clockwise and only after the samples had been properly cooled.

\section{4-Data analysis, model validation and model comparison}

212 The studied coatings where analysed by means of the following metrics [68]:

213

$$
g=g_{s}+\frac{\left(g_{s a t}-g_{s}\right) \delta^{2}}{\delta^{2}+C_{B}}
$$

In Eq.28 $C_{B}$, is a material-related constant .The main difference between both models

224 (Eq. 24 and Eq.28) is that the latter tends to a saturation value $g_{\text {sat }}$ gloss whilst the 225 former tends to infinite gloss values. Since gloss cannot increase infinitively (as viscosity can) it will only work for moderately thin layers. As expected, the maximum film

227 thickness $\left(\delta_{\text {sat }}\right)$ upon which the change in gloss will have physical meaning will be: 


$$
\delta_{s a t}=\left(\frac{g_{s a t}}{\wp t_{1}}\right)^{\frac{1}{t_{2}}}
$$

228 And, in turn, the maximum allowable gloss attainable $\left(g_{\text {sat }}\right)$ will be :

$$
g_{\text {sat }}=g_{s}+J\left(\delta_{\text {sat }}\right) \wp \delta_{\text {sat }}^{t_{2}}
$$

229 Eq. 29 and Eq.30 provide interesting tools for comparing and characterising coatings.

$230 \delta_{\text {sat }}$ value may also be of use for assessing the range of validity of non-finite gloss 231 mathematical models ${ }^{8}$. Namely:

$$
\begin{array}{ll}
g=g_{s}+J(\delta) \wp \delta^{t_{2}} & 0 \leq \delta \leq \delta_{\text {sat }} \\
g=g_{\text {sat }} & \delta \geq \delta_{\text {sat }}
\end{array}
$$

232 For each kind of coating the following steps and formulas will be used for parameter 233 estimation. Gloss has been expressed on a relative basis $G_{i}$ (Eq.31) for the calculations 234 involving glossy coatings:

$$
G_{i}=\frac{g_{i}-g_{s}}{g_{s}}
$$

\footnotetext{
${ }^{8}$ This piecewised mathematical formulation is a necessary consequence of using simplistic models.
} 
238 In order to validate the modelling approach presented in the previous sections several

239 datasets have been used. Such datasets cover a wide range of both organic and

240 inorganic substance, either with matting or glossy properties and even measured at

241 different reference angles. The main idea behind the heterogeneity of the data was

242 checking for the eventual limitations one might encounter when using Eq.26 for its

243 description. Fig.4 summarizes for each kind of coating which parameters, measurements

244 and procedures have been implemented.

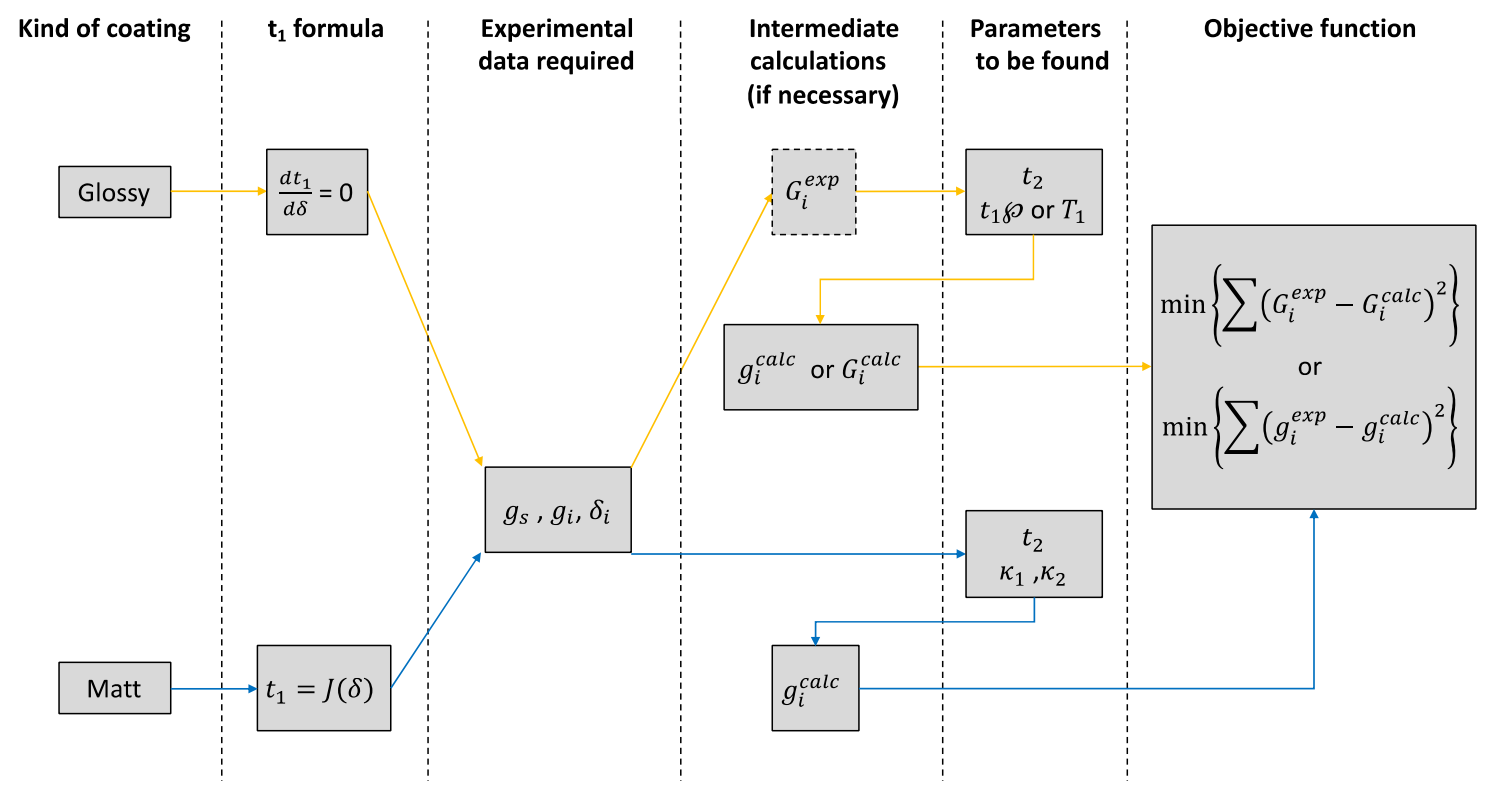

246 Fig4. Flowchart for model parameter estimation

248 Four glossy inorganic coatings (Zinc oxide, zinc oxide + rubber, zinc sulphide and

249 litophone ${ }^{9}$ ), two kinds of organic lacquers, one glossy PUD-base topcoat and three

250 different-weaved silk fabrics have been investigated. Dataset 1 can be found in Table 1.

\footnotetext{
${ }^{9}$ Litophone: A material obtained as a result of the coprecipitation of $\mathrm{BaSO}_{4}$ and $\mathrm{ZnS}$ [73].
} 
252 Two PUD-based matting agents along with two purely inorganic silica coatings of varying 253 particle size and three $\mathrm{PVC}-\mathrm{TiO}_{2}$ films with increasing matting content have been tested.

254 Dataset 2 can be found in Table 2.

255

256

257

258

259

260

261

262

263

264

265

266

267

268

269

270

271

272

273

274

275

276

277

278

279 





Table 2: Film-thickness vs gloss of different matting coatings (Dataset 2).

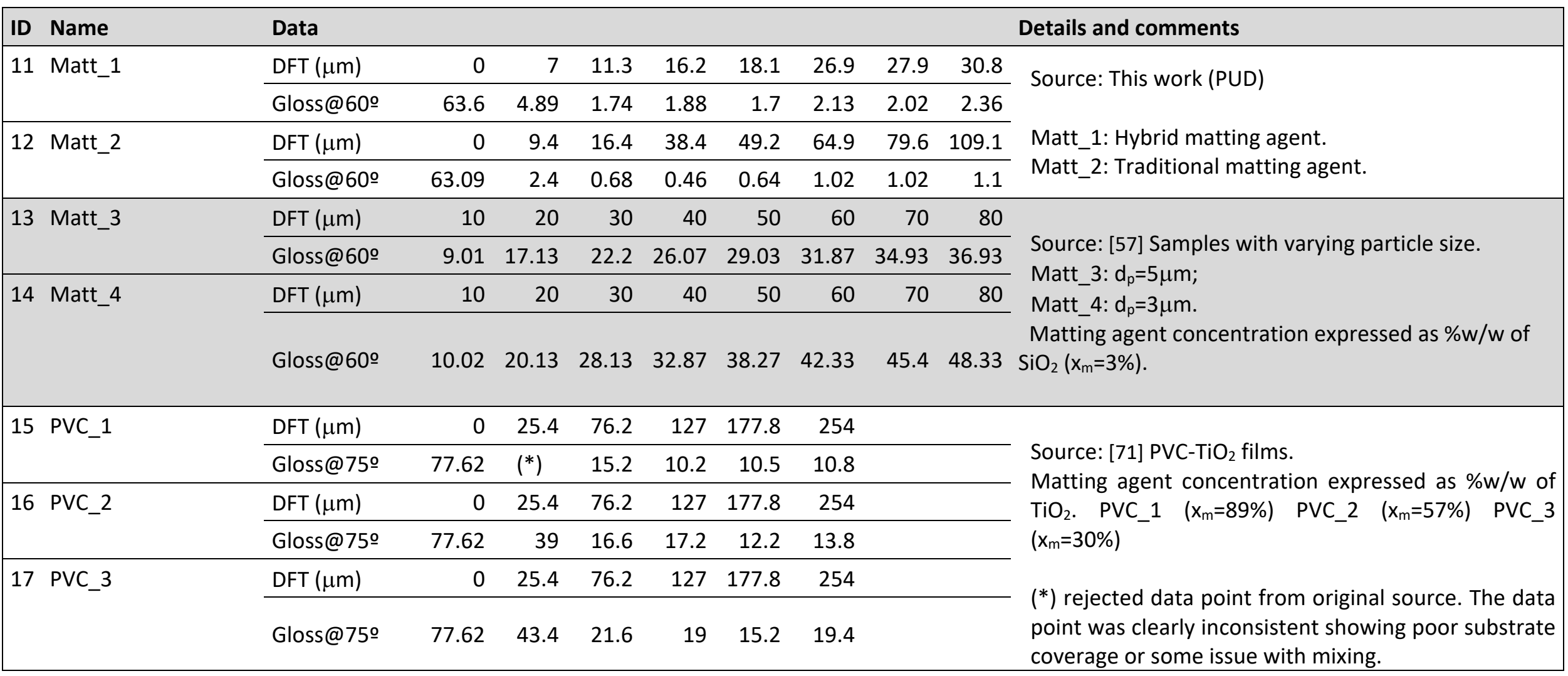


292 The data is plotted in Graphs 2 to 8, along with the corresponding mathematical models 293 forecasts . Fitting results are summarized in tables 2 and 3.

294



Graph 2: Film thickness effect on gloss for several inorganic glossy coatings.



Graph 3: Film thickness effect on gloss for a PUD glossy topcoat. 


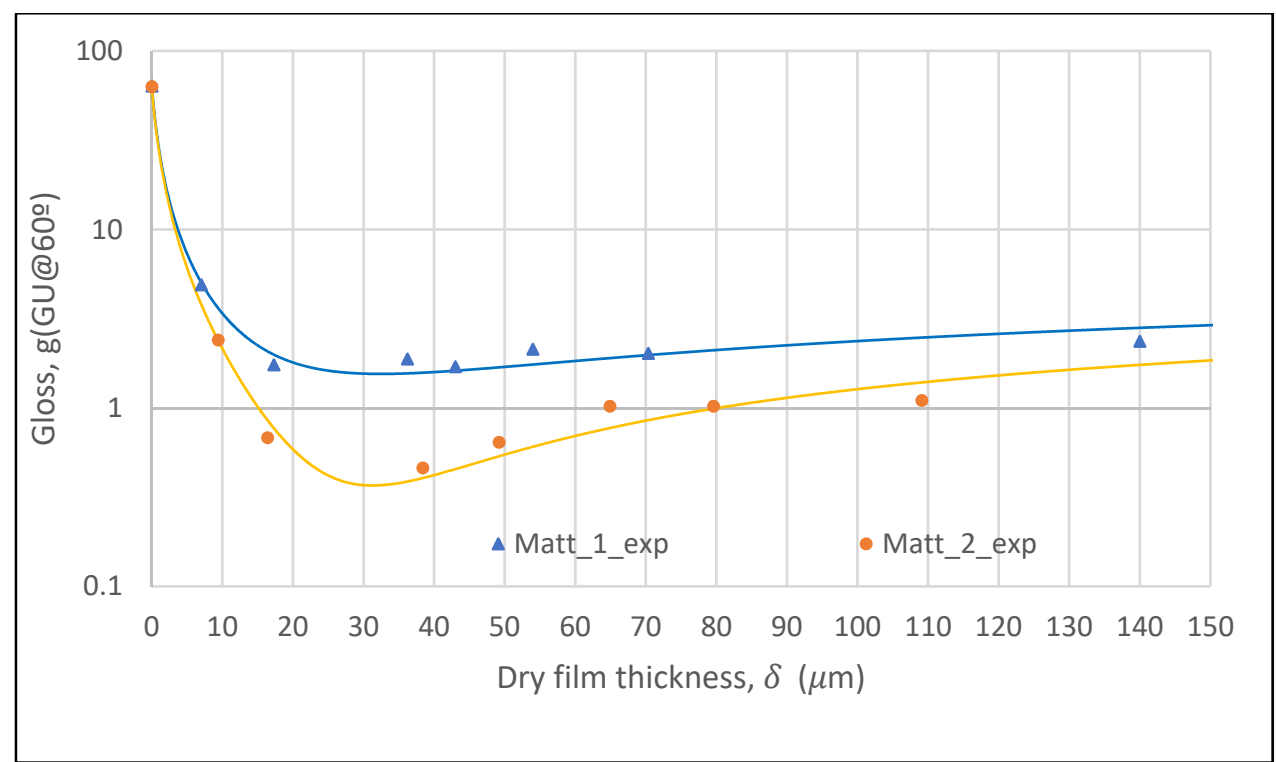

301

Graph 4: Film thickness effect on gloss for matting PUDs

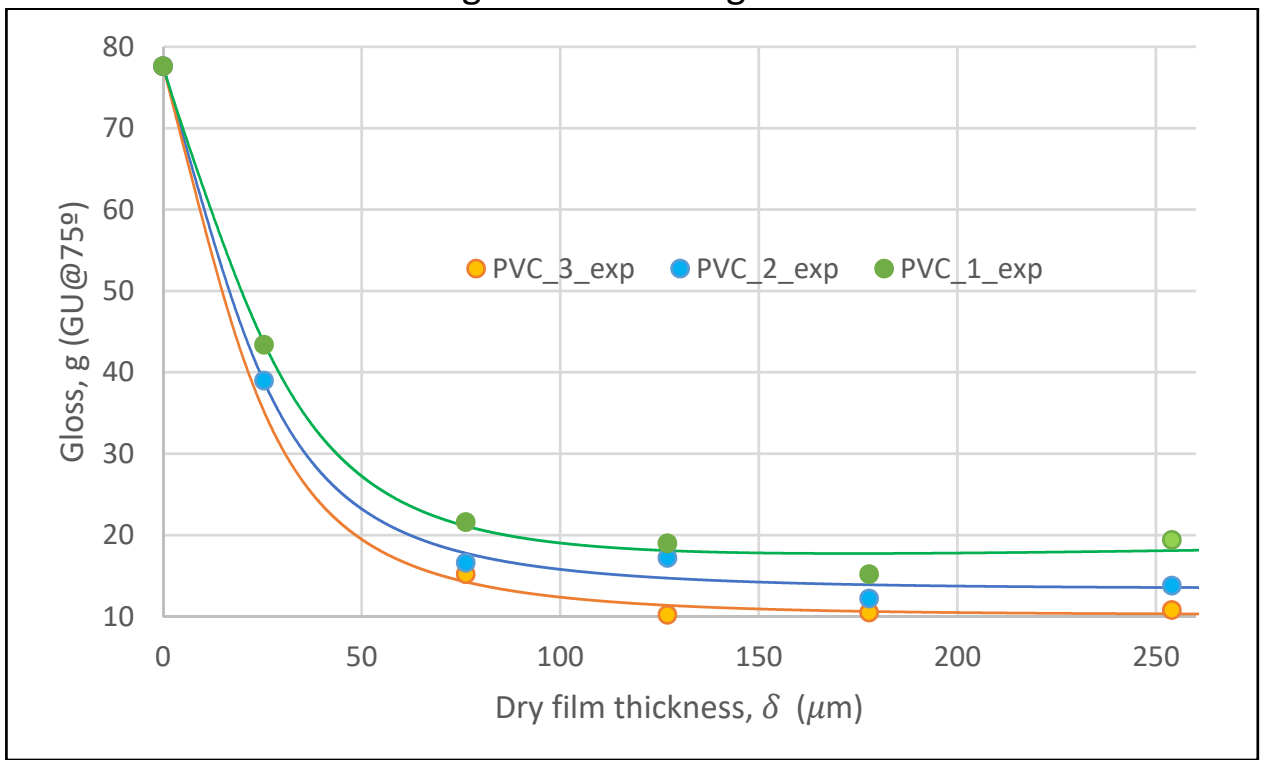

Graph 5: Film thickness effect on gloss for $\mathrm{PVC} / \mathrm{TiO}_{2}$ hybrid matting agents

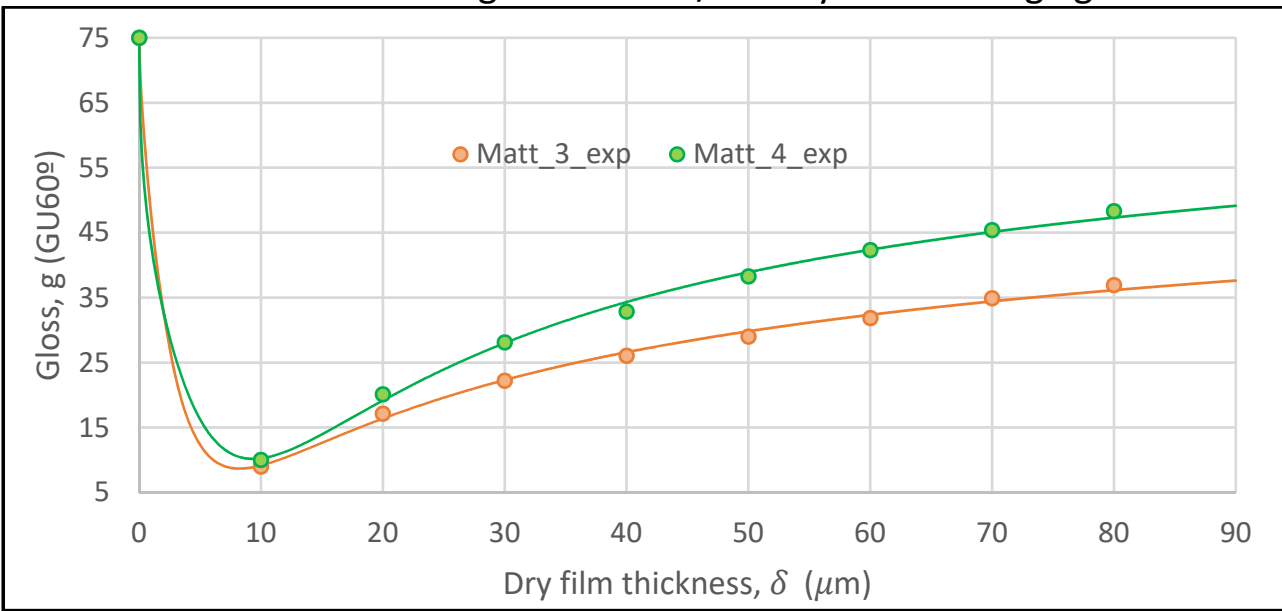




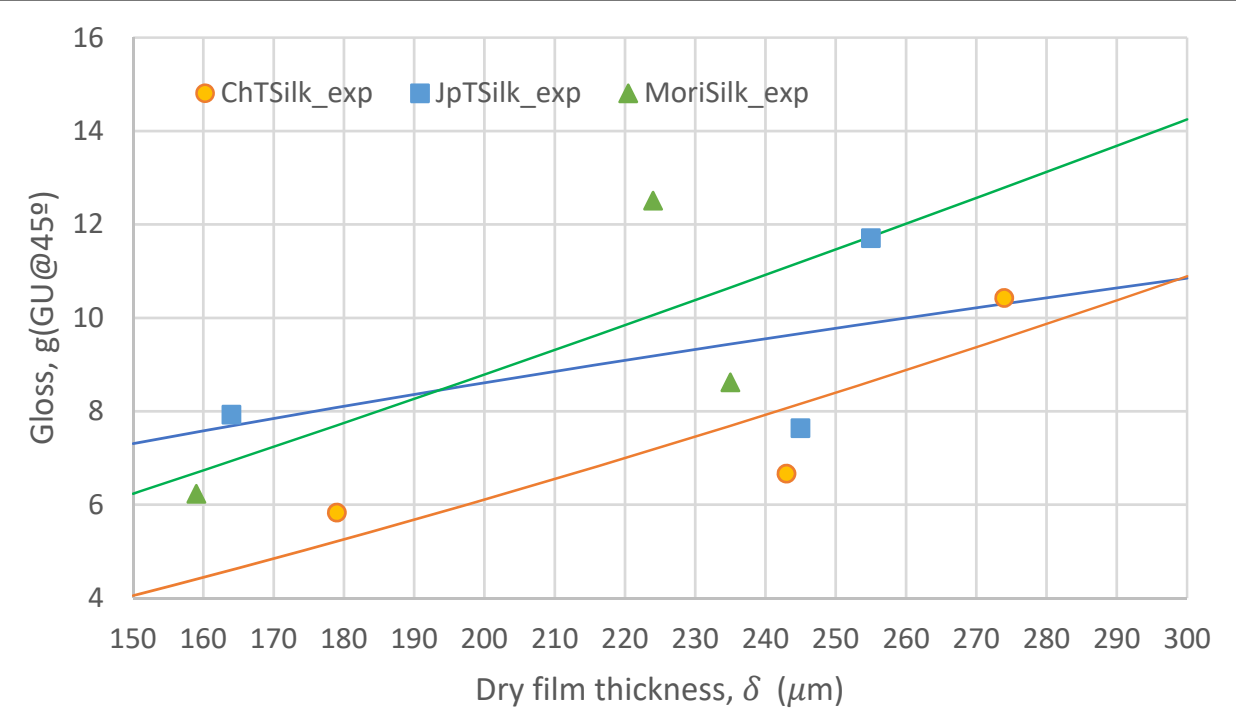

311

Graph 7: Film thickness effect on gloss for several weaved silks.

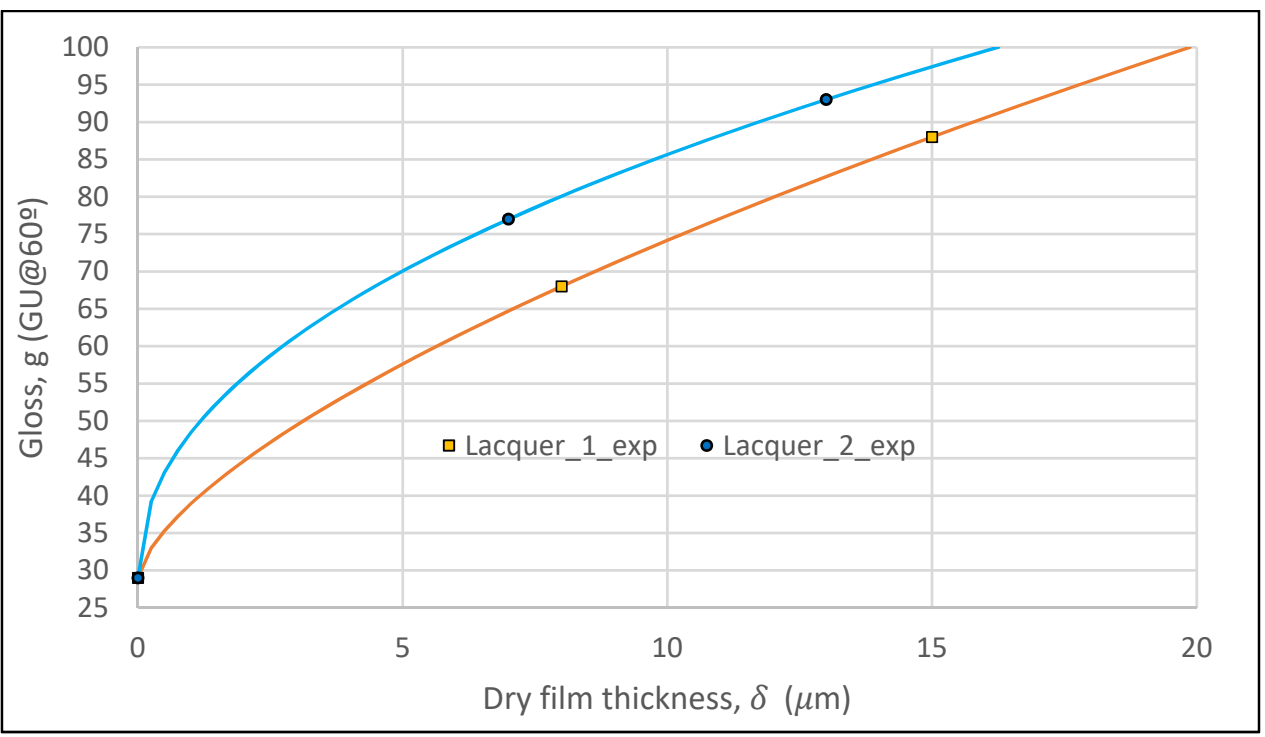

313 Graph 8: Film thickness effect on gloss for lacquers

314

315

316

317

318

319 
Table 2: Metrics for glossy coatings

\begin{tabular}{|c|c|l|c|c|c|c|c|}
\hline ID & Dataset & \multicolumn{1}{|c|}{ Fitted equations } & SSE & $\boldsymbol{R}_{\text {adj }}^{2}$ & Parity equation & $\boldsymbol{\delta}_{\text {sat }}^{\text {calc }}{ }^{10}(\mu \mathrm{m})$ & $\boldsymbol{\delta}_{\text {sat }}^{\text {exp }}{ }^{11}(\boldsymbol{\mu m})$ \\
\hline 1 & Lacquer_1 & $\mathrm{g}=29+9.92 \cdot \delta^{0.66}$ & $10^{-7}$ & 1.0000 & $\mathrm{y}=\mathrm{x}$ & 19.72 & - \\
\hline 2 & Lacquer_2 & $\mathrm{g}=29+19.43 \cdot \delta^{0.46}$ & $10^{-9}$ & 1.0000 & $\mathrm{y}=\mathrm{x}$ & 16.73 & - \\
\hline 3 & Lit & $\mathrm{g}=19.04 \cdot \delta^{0.45}$ & 83.4 & 0.9791 & $\mathrm{y}=2.8785+0.9575 \mathrm{x}$ & 62.22 & 190 \\
\hline 4 & ZnO & $\mathrm{g}=11.2 \cdot \delta^{0.53}$ & 20.19 & 0.9899 & $\mathrm{y}=0.3947+0.9930 \mathrm{x}$ & 87.51 & 152 \\
\hline 5 & ZnORb & $\mathrm{g}=11.69 \cdot \delta^{0.48}$ & 43.6 & 0.9650 & $\mathrm{y}=0.6958+0.9884 \mathrm{x}$ & 37.91 & 56.2 \\
\hline 6 & ZnS & $\mathrm{g}=20.20 \cdot \delta^{0.44}$ & 0.52 & 0.2749 & $\mathrm{y}=6.5422+0.2796 \mathrm{x}$ & - & - \\
\hline 7 & JpTSilk & $\mathrm{g}=0.42 \cdot \delta^{0.57}$ & 0.86 & 0.7456 & $\mathrm{y}=1.6565+0.7805 \mathrm{x}$ & - & - \\
\hline 8 & ChTSilk & $\mathrm{g}=0.003 \cdot \delta^{1.43}$ & 0.69 & 0.4860 & $\mathrm{y}=4.8396+0.4705 \mathrm{x}$ & - & - \\
\hline 9 & MoriSilk & $\mathrm{g}=0.016 \cdot \delta^{1.19}$ & 0.26 & 0.9997 & $\mathrm{y}=0.0093+0.9996 \mathrm{x}$ & 289.6 & - \\
\hline 10 & Glossy_1 & $\mathrm{g}=61.16+12.5 \cdot \delta^{0.2}$ & & & & - \\
\hline
\end{tabular}

the model does no accurately fit experimental data and consequently the model parameters lack physical meaning.

${ }^{10}$ Assuming $g_{\text {sat }}$ is $100 \%$ GU@ @ calculated by means of Eq.30.

${ }^{11}$ From [56]. 
Table 3: Metrics for matting coatings

326

\begin{tabular}{|c|c|l|c|c|c|c|c|}
\hline ID & Dataset & Fitted equations & SSE & \multicolumn{1}{|c|}{$\boldsymbol{R}_{\text {adj }}^{2}$} & Parity equation & $\boldsymbol{\delta}^{*}$ & $\mathbf{g}_{\min }$ \\
\hline 11 & Matt_1 & $\mathrm{g}=63.6-227.2 \cdot \tanh \left(\frac{0.31}{1+\delta}\right) \cdot \delta^{0.970}$ & 0.54 & 0.9999 & $\mathrm{y}=0.001+1.0000 \mathrm{x}$ & 32.5 & 1.55 \\
\hline 12 & Matt_2 & $\mathrm{g}=63.1-192.7 \cdot \tanh \left(\frac{0.37}{1+\delta}\right) \cdot \delta^{0.969}$ & 0.19 & 0.9999 & $\mathrm{y}=0.001+0.9990 \mathrm{x}$ & 31.3 & 0.37 \\
\hline 13 & Matt_3 & $\mathrm{g}=72-27.5 \cdot \tanh \left(\frac{6.47}{1+\delta}\right) \cdot \delta^{0.656}$ & 0.25 & 0.9936 & $\mathrm{y}=0.817+0.9811 \mathrm{x}$ & 8.3 & 8.67 \\
\hline 14 & Matt_4 & $\mathrm{g}=72-32.1 \cdot \tanh \left(\frac{12.49}{1+\delta}\right) \cdot \delta^{0.395}$ & 0.99 & 0.9974 & $\mathrm{y}=0.3058+0.9919 \mathrm{x}$ & 9.4 & 10.18 \\
\hline 15 & PVC_1 & $\mathrm{g}=77.62-1.96 \cdot \tanh \left(\frac{37.17}{1+\delta}\right) \cdot \delta^{0.987}$ & 2.50 & 0.9968 & $\mathrm{y}=0.003+0.9992 \mathrm{x}$ & 309.2 & 10.29 \\
\hline 16 & PVC_2 & $\mathrm{g}=77.62-1.76 \cdot \tanh \left(\frac{39.57}{1+\delta}\right) \cdot \delta^{0.985}$ & 10.5 & 0.9968 & $\mathrm{y}=0.102+0.9964 \mathrm{x}$ & 323.5 & 13.51 \\
\hline 17 & PVC_3 & $\mathrm{g}=77.62-1.69 \cdot \tanh \left(\frac{48.93}{1+\delta}\right) \cdot \delta^{0.944}$ & 3.09 & 0.9939 & $\mathrm{y}=-0.668+1.014 \mathrm{x}$ & 177.0 & 17.4 \\
\hline
\end{tabular}


328 Regarding what was presented in Eq. 13 several expressions were tested for modeling

$329 t_{1}=J(\delta)$. Some of the main requirements for the mathematical model were to include

330 a hyperbolic trigonometric function and to predict a nonnegative minimum gloss value.

331 Eq. 21 shows a suitable expression for $t_{1}$ :

$$
t_{1}=\kappa_{0} \tanh \left(\frac{\kappa_{2}}{\delta+1}\right)
$$

332 Where $\kappa_{0}<0$. When the aforementioned equation is plugged in Eq.14 the GFTR for 333 matting agents is finally devised:

$$
\mathrm{g}=\mathrm{g}_{\mathrm{s}}+\kappa_{1} \delta^{t_{2}} \tanh \left(\frac{\kappa_{2}}{\delta+1}\right)
$$

334 where $\kappa_{0} \wp=\kappa_{1}$. If $\frac{d g}{d \delta}$ is set to 0 it is found that $\delta^{*}=f\left(t_{2}, \kappa_{2}\right)$. For the sake of simplicity 335 only the results found using Eq.32 will be presented and further treatment of this section 336 will be developed in the discussion.

337

338 5-Discussion

339 5.1-General aspects

340 The goodness of fit of all mathematical models is high in regard of the different statistical

341 tests performed. High correlation coefficients values along with randomly distributed

342 residuals (calculated but not shown), symmetric parity charts and low SSE asses the 343 usefulness of the equations presented in this contribution. The only systems which are 344 not well described by the mathematical models are those involving fabrics. 


$$
\kappa_{1}=\kappa_{0}\left(\frac{\varphi_{f} w_{M A, f}}{\alpha \delta_{0} \varphi_{0} w_{M A, 0}}\right)^{t_{2}}
$$

347 if Eq.34 is linearized it can be shown that $\ln \left(-\kappa_{1}\right) \propto-\mathrm{t}_{2} \ln \left(w_{M A, 0}\right)$. Plotting the results obtained for after fitting $\kappa_{1}$ for the PVC_1, 2 and 3 datasets confirms the model validity in accounting for varying matting agent/matting polymer concentration:

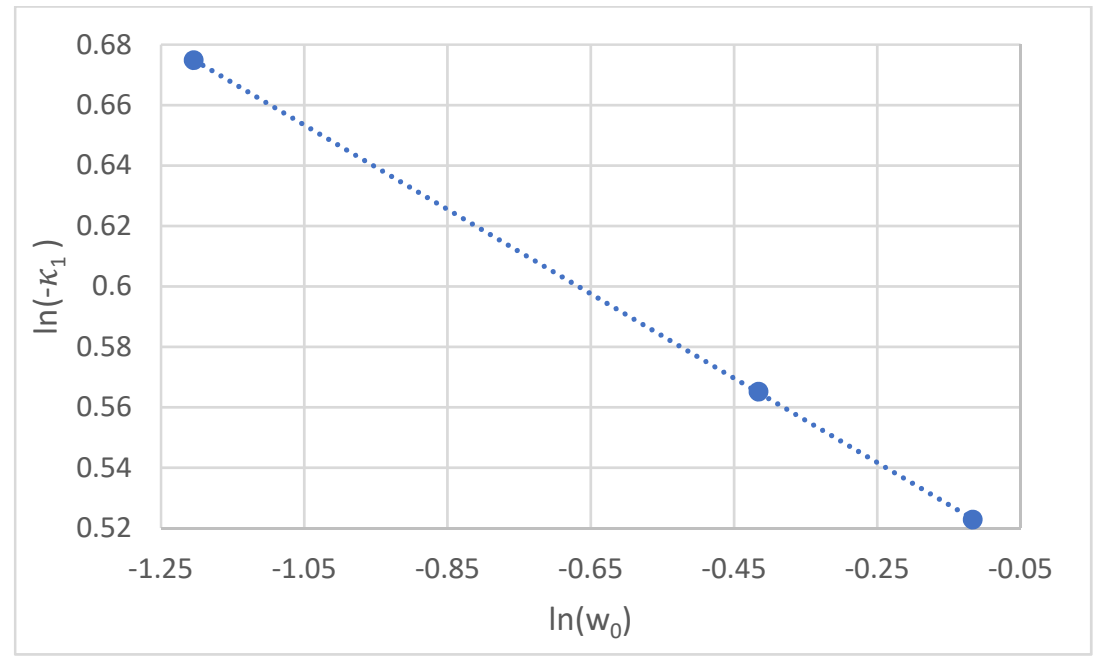

351 Fig. 6: Functional dependence of model parameters with matting agent concentration.

\section{3-Prediction of minimum gloss,$\delta^{*}$ and $\delta_{\text {sat }}$}

353 For a given matting agent its matting efficiency could be related to its minimum gloss. A

354 more efficient matting agent would require a smaller amount of coating so as to obtain

355 its minimum gloss. Despite this, experimental results do not provide conclusive results 356 in that regard.

357 The models undercalculate saturation film thickness values. This is probably due to the 358 fact that the original model does not account for that saturation behaviour. 
363 After fitting the equations it became evident that whilst most of glossy coatings had a power value between 0.5 and 0.7 as predicted by our modeling approach the matting agents power value clustered around unity. This difference is most likely due to the fact that the presence of matting agents (polymers/particles) rendered appreciable alteration in film forming dynamics. Surface tension acts as a driving force for levelling and plays an important part in reducing film area. According to this, for a coating with hindered film formation, effective surface tension will be lower than expected. The film forming driving force can be expressed as the difference between two Ohnesorge numbers. Additionally, if $t_{2} \approx 1$ it becomes evident that:

$$
\Delta \sigma \propto \Delta\left(\delta^{\frac{1-t_{2}}{t_{2}}}\right) \rightarrow \Delta \sigma \approx 0
$$

372 Therefore, for matting agents (as expected), not so smooth films are obtained yielding 373 rough surfaces. The rheologic behaviour of the coatings will also play an important role providing stresses are involved.

\section{6-Conclusions and future work}

376 Gloss and film thickness for several kinds of glossy and matt coatings have been

377 successfully correlated by means of easy-to-use models. The model derivation shows consistency with important process variables such as matting agent concentration (as seen in Fig.6). Glossy coatings can be represented by a power-like model where the power value lies closer to $0.5-0.7$ for Newtonian coatings. For non-Newtonian glossy coatings the power value lies far from that interval showing strong deviations. Such deviations where expectable given the fact that the new expression derived for $n=2$ was based upon levelling time and momentum transference equation in which Newtonian 
arguments where used. Interferences with surface tension levelling due to the presence

385

of matting agents have been also considered. The matting coatings follow a hyperbolically modified power-law (loosely similar to Kubelka-Munk expressions) which is able of predicting a minimum gloss value as it is found empirically.

Both presented models account for the substrate gloss (i.e. they meet the no coating border condition) but since they are derived from a non-finite viscosity model they tend to infinite gloss values instead of showing a saturation behaviour. Subsequently, a piecewise function is required. $\delta_{\text {sat }}$ estimation is currently impossible as well. Other surface defects such as micro-sagging or micro-bubbling cannot be accounted by the mathematical model despite its relevance requiring specific mathematical treatment. The case where a substantial degree of texture and optical isotropy (birefringence, etc.) is found highlights another of the model's main limitations. For those cases, the model fails in reproducing experimental data.

Future work concerning the present study should certainly deal with:

- A deeper exploration of the properties of the nondimensional equation $O h=$ $f(B o, C a)$ and verifying its applicability in scale up /modeling. .

- A careful study of the influence of material properties such as density or viscosity in model parameters such as saturation or minimum gloss and film thicknesses.

\section{References}

[1] J. Uribe-padilla, J. Salgado-valle, and M. Graells-Sobrè, "A novel contribution to the modeling of the matting efficiency of aqueous polyurethane dispersions," Prog. Org. Coatings, 2017.

[2] L. A. Simpson, "Factors controlling gloss of paint films," Prog. Org. Coatings, vol. 6, no. 1, pp. 1-30, 1978.

[3] Z. W. Wicks, F. N. Jones, S. P. Pappas, and D. A. Wicks, Organic coatings: science and technology, Third edition. 2007. 
412 [5] F. Martin, P. Muralt, M.-A. Dubois, and A. Pezous, "Thickness dependence of the properties of highly $c$-axis textured AIN thin films," J. Vac. Sci. Technol. A Vacuum, Surfaces, Film., 2004.

[6] Y. Zhao, A. M. Nardes, and K. Zhu, "Solid-state mesostructured perovskite $\mathrm{CH} 3 \mathrm{NH} 3 \mathrm{Pbl} 3$ solar cells: Charge transport, recombination, and diffusion length," J. Phys. Chem. Lett., 2014.

[7] B. Tlili, C. Nouveau, M. J. Walock, M. Nasri, and T. Ghrib, "Effect of layer thickness on thermal properties of multilayer thin films produced by PVD," Vacuum, 2012.

[8] S. Mali, M. V. E. Grossmann, M. A. García, M. N. Martino, and N. E. Zaritzky, "Mechanical and thermal properties of yam starch films," Food Hydrocoll., 2005.

[9] G. Skordaris et al., "Film thickness effect on mechanical properties and milling performance of nano-structured multilayer PVD coated tools," Surf. Coatings Technol., 2016.

[10] K. D. Bouzakis et al., "The effect of coating thickness, mechanical strength and hardness properties on the milling performance of PVD coated cemented carbides inserts," Surf. Coatings Technol., 2004.

[11] J. H. Kuang and H. L. Chien, "The effect of film thickness on mechanical properties of TiN thin films," Adv. Sci. Lett., 2011.

[12] M. Buntinx et al., "Evaluation of the thickness and oxygen transmission rate before and after thermoforming mono- and multi-layer sheets into trays with variable depth," Polymers (Basel)., 2014.

[13] C. Charton, N. Schiller, M. Fahland, A. Holländer, A. Wedel, and K. Noller, "Development of high barrier films on flexible polymer substrates," in Thin Solid Films, 2006.

[14] B. Bravin, D. Peressini, and A. Sensidoni, "Development and application of polysaccharide-lipid edible coating to extend shelf-life of dry bakery products," J. Food Eng., 2006.

[15] A. Kamel, D. Pascal, B. Isabelle, and L. Pierre, "Model predictive control of a powder coating curing process: Application of the MPC@CB software," in Proceedings of the 26th Chinese Control Conference, CCC 2007, 2007.

[16] R. Turton, "The application of modeling techniques to film-coating processes Film coating," Drug Development and Industrial Pharmacy. 2010.

[17] D. He, Z. Wang, L. Yang, and Z. Mao, "Optimization control of the color-coating production process for model uncertainty," Comput. Intell. Neurosci., vol. 2016, 2016.

[18] T. M. Sullivan and S. Middleman, "Film thickness in blade coating of viscous and viscoelastic liquids," J. Nonnewton. Fluid Mech., vol. 21, no. 1, pp. 13-38, 1986.

[19] I. Iliopoulos and L. E. Scriven, "A blade-coating study using a finite-element simulation," Phys. Fluids, 2005.

[20] R. W. Hewson and N. Kapur, "Effects of shear thinning on forward roll coating," Chem. Eng. Res. Des., 2013.

[21] G. A. Zevallos, M. S. Carvalho, and M. Pasquali, "Forward roll coating flows of viscoelastic liquids," J. Nonnewton. Fluid Mech., 2005.

[22] E. V. A. N. M. Itsoulis and G. E. A. Thanasopoulos, "NUMERICAL SIMULATION OF BLADE-OVER-ROLL COATING," Comput. methods Mater. Sci., 2010. 
482

483

484

485

486

487

488

489

490

491

492

493

494

495

496

497

498

499

500

501

502

503

504
[23] A. Javadi, H. S. Mehr, M. Sobani, and M. D. Soucek, "Cure-on-command technology: A review of the current state of the art," Prog. Org. Coatings, 2016.

[24] M. Nabil and A. S. Rattner, "A Computational Study on the Effects of Surface Tension and Prandtl Number on Laminar-Wavy Falling-Film Condensation," J. Heat Transfer, 2017.

[25] D. Grosso, "How to exploit the full potential of the dip-coating process to better control film formation," J. Mater. Chem., 2011.

[26] X. Zhang, "Several fundamental researches on structural integrity of plasmasprayed coating-based systems," Weld. World, 2013.

[27] M. Javidi, M. A. Pope, and A. N. Hrymak, "Investigation on dip coating process by mathematical modeling of non-Newtonian fluid coating on cylindrical substrate," Phys. Fluids, 2016.

[28] L. Xu, X. Li, Y. Chen, and F. Xu, "Structural and optical properties of ZnO thin films prepared by sol-gel method with different thickness," Appl. Surf. Sci., 2011.

[29] J. P. Enríquez and X. Mathew, "Influence of the thickness on structural, optical and electrical properties of chemical bath deposited CdS thin films," Sol. Energy Mater. Sol. Cells, 2003.

[30] S. Y. Kim, "Simultaneous determination of refractive index, extinction coefficient, and void distribution of titanium dioxide thin film by optical methods.," Appl. Opt., 1996.

[31] Q. Yong et al., "Synthesis and surface analysis of self-matt coating based on waterborne polyurethane resin and study on the matt mechanism," Polym. Bull., pp. 1-16, 2016.

[32] A. Goldschmidt and H.-J. Streitberger, "Basics of coating technology," 2007, p. 30.

[33] C. Francis, "Thickness of a Film Liquid Adhering to a Surface Slowly Withdrawn from the Liquid," NIST, vol. 25, 1940.

[34] A. W. Crook, "The Lubrication of Rollers II. Film Thickness with Relation to Viscosity and Speed," Philos. Trans. R. Soc. A Math. Phys. Eng. Sci., vol. 254, no. 1040, pp. 223-236, 1961.

[35] B.Derjaguin, "On the Thickness of the Liquid Film Adhering to the Walls of a Vessel after Emptying," Prog. Surf. Sci., vol. 43, no. 1-4, pp. 134-137, 1993.

[36] L. Cisneros-Zevallos and J. M. Krochta, "Dependence of Coating Thickness on Viscosity of Coating Solution Applied to Fruits and Vegetables by Dipping Method," Food Eng. Phys. Prop. Depend., vol. 68, no. 2, 2003.

[37] A. Lee, P.-T. Brun, J. Marthelot, G. Balestra, F. Gallaire, and P. M. Reis, "Fabrication of slender elastic shells by the coating of curved surfaces," Nat. Commun., vol. 7, pp. 1-7, 2015.

[38] S. D. R. Wilson, "The drag-out problem in film coating theory," J. Eng. Math., vol. 16, no. 3, pp. 209-221, 1982.

[39] L. Landau and B. Levich, "Dragging of a Liquid by a Moving Plate," Acta Physicochim. U.R.S.S., vol. 17, no. 1-2, pp. 42-54, 1942.

[40] H. N. Dixit and G. M. Homsy, "The elastocapillary Landau-Levich problem," J. Fluid Mech., vol. 735, pp. 1-28, 2013.

[41] R. R. Eley, "Applied Rheology in the Protective and Decorative Coatings Industry," Rheol. Rev., vol. 3, pp. 173-240, 2005.

[42] H. Zhai, "Effect of Film Thickness on the Rheological Behaviors of Asphalt 
Binders," Asphalt, no. 001373, pp. 7-14, 2000.

[43] M. A. and K. K. Shin Kasahara, Hirota Saito, Makoto Hosotani, Noriko Hatakeyama, "Relation between Viscosity and Film Thickness of Dental Luting Cements," Prosthodontics, 2001.

[44] S. Wu, L. Pang, G. Liu, and J. Zhu, "Laboratory Study on Ultraviolet Radiation Aging of Bitumen," J. Mater. Civ. Eng., vol. 22, no. August, pp. 767-772, 2010.

[45] A. F. Routh, "Drying of thin colloidal films.," Rep. Prog. Phys., vol. 76, no. 4, p. 046603, 2013.

[46] C. Petersen, C. Heldmann, and D. Johannsmann, "Internal stresses during film formation of polymer latices," Langmuir, vol. 15, no. 11, pp. 7745-7751, 1999.

[47] T. Ismail, Modeling in Transport Phenomena, no. October. 2009.

[48] I. Ludwig, W. Schabel, P. Ferlin, J. C. Castaing, and M. Kind, "Drying, film formation and open time of aqueous polymer dispersions," in European Physical Journal: Special Topics, 2009.

[49] A. Tracton, COATINGS TECHNOLOGY FUNDAMENTALS, TESTING, AND PROCESSING TECHNIQUES. 2007.

[50] R. Keunings and D. W. Bousfield, "Analysis of Surface Tension Driven Leveling in Viscoelastic Films," J. Non-Newtonian Fluid Mech. Elsevier Sci. Publ. B.V, vol. 22, pp. 219-233, 1987.

[51] H. S. Kheshgi and L. E. Scriven, "The evolution of disturbances in horizontal films," Chem. Eng. Sci., vol. 43, no. 4, pp. 793-801, 1988.

[52] L. E. Rodd, T. P. Scott, J. J. Cooper-White, and G. H. McKinley, "Capillary breakup rheometry of low-viscosity elastic fluids," Appl. Rheol., vol. 15, no. 1, pp. 1227, 2005.

[53] S. J. Jeon, "Mechanisms of Print Gloss Development with Controlled Coating Structure," 2002.

[54] P.-G. de Gennes, F. Brochard-Wyart, and D. Quéré, Capillarity and Wetting Phenomena. 2004.

[55] G. H. McKinley, "Dimensionless groups for understanding free surface flows of complex fluids," Soc. Rheol. Bull., vol. July, no. 05, p. 8, 2005.

[56] A.H.Pfund, "A relation between the Brightness and hidding power of white-paint pigments," Ind. Eng. Chem., 1930.

[57] J.Bieleman, Additives for Coatings, vol. 41, no. 1-3. 2001.

[58] G. P. Bierwagen, "Estimation of film thickness nonuniformity effects on coating optical properties," Color Res. Appl., vol. 17, no. 4, pp. 284-292, 1992.

[59] A. Goldschmidt and H.-J. Streitberger, Basics of coating technology. 2007.

[60] T. E. Fletcher, "A simple model to describe relationships between gloss behaviour, matting agent concentration and the rheology of matted paints and coatings," Prog. Org. Coatings, vol. 44, no. 1, pp. 25-36, 2002.

[61] T. J. Dougherty and I. M. Krieger, "Potential Around a Charged Colloidal Sphere," J. Phys. Chem., vol. 63, no. 11, pp. 1869-1872, 1959.

[62] W. Wendlandt and H. Hecht, Reflectance Spectroscopy. 1966.

[63] C. Wiener, "Die Zerstreuung des Lichtes durch matte Oberflächen," Ann. Phys., vol. 283, no. 12, pp. 638-658, 1892.

[64] J. V. KOLESKE, Paint and Coating Testing Manual: 15th edition of the GardnerSward handbook. 2012.

[65] L. Gamble and A. H. Pfund, "Experimental Determination of Brightness-Film 
Thickness Curves of," Ind. Eng. Chem., pp. 63-66, 1930.

[66] P. G. Judd DB, Harrison WN, Sweo BJ, Hickson EF, Eickhoff AJ, Shaw MB, "Optical Specification of Light*scattering Materials," J Res Nat Bur Stand, vol. 19, pp. 287-317, 1937.

[67] H. D. Bruce, A photometric method for measuring the hiding power of paints, vol. 20, no. no 306. 1926.

[68] G. E. P. Box, J. S. Hunter, and W. G. Hunter, "Statistics for Experimenters," in Statistics for Experimenters: Design, Innovation and Discovery, 2005.

[69] R. Lu and T. Miyakoshi, Lacquer Chemistry and Applications. 2015.

[70] H. Kato, T. Hata, and T. Takahashi, "Characteristics of wild silk fibers and processing technology for their use," Japan Agric. Res. Q., vol. 31, no. 4, pp. 287-294, 1997.

[71] G. H. Al-Ghamdi, E. D. Sudol, V. L. Dimonie, and M. S. El-Aasser, "High PVC filmforming composite latex particles via miniemulsification, Part 1: Preparation," J. Appl. Polym. Sci., vol. 101, no. 6, pp. 4504-4516, 2006.

[72] D. B. Hall, P. Underhill, and J. M. Torkelson, "Spin coating of thin and ultrathin polymer films," Polym. Eng. Sci., 1998.

[73] H. G. Völz, "Pigments, Inorganic, 1. General," in Ullmann's Encyclopedia of Industrial Chemistry, 2012, pp. 225-256. 